\title{
Source of magnetic field effects on the electrocatalytic reduction of $\mathrm{CO}_{2}$
}

\author{
Thomas C. Player and P. J. Hore
}

Department of Chemistry, University of Oxford, Physical and Theoretical Chemistry Laboratory, Oxford, United Kingdom

*Author for correspondence: peter.hore@,chem.ox.ac.uk

\section{ABSTRACT}

We present an analysis of reported magnetic field effects on the yield of formic acid produced by electrocatalytic reduction of carbon dioxide at a nanoparticle tin electrode (Pan et al., J. Phys. Chem. Lett. 11 (2020) 48-53). Radical pair spin dynamics simulations are used to show that: (1) the $\Delta g$ mechanism favoured by Pan et al. is not sufficient to explain the observed magneto-current. (2) Field-dependent spin relaxation, resulting from the anisotropy of the $g$-tensor of $\mathrm{CO}_{2}^{\circ-}$, combined with the coherent singlet-triplet interconversion arising from isotropic hyperfine and Zeeman interactions, can quantitatively account for the observed magnetic field effect. (3) Modification of hyperfine interactions by isotopic substitution $\left({ }^{1} \mathrm{H} \rightarrow{ }^{2} \mathrm{H}\right.$ and $/$ or $\left.{ }^{12} \mathrm{C} \rightarrow{ }^{13} \mathrm{C}\right)$ could be used to test both the proposed reaction mechanism and the interpretation presented here.

\section{INTRODUCTION}

Conversion of carbon dioxide into small organic molecules is appealing as a way to reduce the accumulation of atmospheric $\mathrm{CO}_{2}$ and to generate useful chemicals. ${ }^{1-7}$ Pan et al. recently reported that the yield of formic acid in the electrocatalytic reduction of $\mathrm{CO}_{2}$ at a nanoparticle tin electrode could be increased by up to $100 \%$ by application of a $900 \mathrm{mT}$ magnetic field. ${ }^{8}$ The authors rationalised this observation as a change in the efficiency of interconversion of the electronic singlet and triplet states of a radical pair comprised of a $\mathrm{CO}_{2}^{--}$radical and a hydrogen atom $\left(\mathrm{H}^{\circ}\right)$ 
formed by electroreduction of $\mathrm{CO}_{2}$ and bicarbonate $\left(\mathrm{HCO}_{3}^{-}\right)$, respectively. Figure 1(a) shows the proposed reaction scheme. ${ }^{8}$ The radical pairs are formed in a statistical 3:1 mixture of triplet (total electron spin, $S=1$ ) and singlet $(S=0)$. Spin selection rules allow singlet, but not triplet, pairs to combine to produce the diamagnetic formate ion, $\mathrm{HCO}_{2}^{-}$. In parallel, the radicals can separate by translational diffusion without reacting. Such "F-pairs" can be sensitive to applied magnetic fields even though they lack the initial spin coherence of the pure singlet or pure triplet states that are usually formed in photochemical reactions. Spin coherence arises in F-pairs from asymmetric reactivity of the singlet and triplet states. The radical pair mechanism provides a solid theoretical basis for quantitative analysis of the quantum spin dynamics that underlie these magnetic field effects. $^{9-14}$

Figure 1(b) shows the change in the electrocatalytic current for $\mathrm{CO}_{2}$ reduction (referred to as the magneto-current) measured by varying the strength of an applied magnetic field between 0 and $900 \mathrm{mT}$ in a saw-tooth fashion with a period of $55 \mathrm{~s}^{8}{ }^{8}$ Peak magneto-currents of 42,58 and $90 \%$ were reported for $0.1,0.2$ and $0.3 \mathrm{M} \mathrm{CO}_{2}$-saturated aqueous solutions of $\mathrm{KHCO}_{3}$ when subjected to a $900 \mathrm{mT}$ magnetic field. The corresponding figures for the increase in the yield of formic acid were 40, 57 and 100\%. The sigmoidal shape of Fig. 1(b), with the magnetic field effect tending to saturate at high field, is typical of radical pair reactions. ${ }^{9,15,16}$ The increase in magneto-current and in the amount of formic acid are consistent with a field-induced increase in the efficiency of conversion of triplet pairs into singlets which then react to give $\mathrm{HCO}_{2}^{-}$. Pan et al. ${ }^{8}$ argued that the magnetic sensitivity was due to a combination of two well-established mechanisms for singlettriplet interconversion: (a) the $\Delta g$ mechanism, ${ }^{13,17}$ arising from the difference in the electron Zeeman interactions of the two radicals and (b) the hyperfine mechanism ${ }^{13,17}$ resulting from the magnetic coupling of the electron and the nucleus in $\mathrm{H}^{\bullet}$. Since the former is expected to become more efficient, and the latter less efficient, at high field, Pan et al. argued that the former must be dominant.

However, there are other interactions that could affect the way that $\left[\mathrm{H}^{\bullet} \mathrm{CO}_{2}^{\bullet-}\right]$ radical pairs respond to an applied magnetic field. Rotational modulation of the anisotropic Zeeman interaction of $\mathrm{CO}_{2}^{\bullet-}{ }^{18-20}$ would lead to electron spin relaxation at a rate that increased quadratically with the 
strength of the external field. Enhanced spin relaxation could also result from the spin-rotation interaction in $\mathrm{CO}_{2}^{--} 18,21,22$ and from translational modulation of the exchange interaction of the two radicals. ${ }^{15,23}$

Here we present the results of spin dynamics simulations of the magnetic sensitivity of $\left[\mathrm{H}^{\bullet} \mathrm{CO}_{2}^{\bullet-}\right]$ radical pairs. This study has three aims: (1) to test the assumption that the $\Delta g$ mechanism can account for the observed magnetic field effects; (2) to explore the role of spin relaxation; (3) and to see whether isotopic substitution $\left({ }^{1} \mathrm{H} \rightarrow{ }^{2} \mathrm{H}\right.$ and/or $\left.{ }^{12} \mathrm{C} \rightarrow{ }^{13} \mathrm{C}\right)$ could offer additional insight into the mechanism of the reaction and the source of the magnetic field effect.

\section{METHODS}

The fraction of $\left[\mathrm{H}^{\bullet} \mathrm{CO}_{2}^{\bullet-}\right]$ radical pairs that combine to produce formic acid was calculated by numerical solution of a stochastic Liouville equation for the density matrix of a spin system comprising the electron and proton in $\mathrm{H}^{\bullet}$ and the unpaired electron in $\mathrm{CO}_{2}^{--}$. Full details are given in the Supplementary Material. The coherent spin dynamics of the radical pair were modelled by means of a spin Hamiltonian, $H$, containing the isotropic Zeeman interactions of the two electron spins with the applied magnetic field and the isotropic hyperfine interaction in $\mathrm{H}^{\bullet}\left(a_{\mathrm{H}}=50.74\right.$ $\mathrm{mT}^{24}$ ). The Zeeman interactions depend on the strength of the applied field, $B$, and the $g$-values of the two radicals $\left(g_{\mathrm{H}}=2.0114,{ }^{25} g_{\mathrm{CO}_{2}}=2.0007^{26}\right)$. Four assumptions were made: (1) that $\mathrm{CO}_{2}^{\circ-}$ tumbles sufficiently rapidly that no coherent spin evolution arises from the anisotropic components of its $g$-tensor; (2) that the average separation of the two diffusing radicals is large enough that there is no net effect of electron spin coupling (i.e. exchange and dipolar interactions) on the coherent spin dynamics; (3) that the nuclear Zeeman interaction in $\mathrm{H}^{\bullet}$ is negligible by virtue of the much smaller magnetic moment of the proton compared to the electron; and (4) that the ${ }^{13} \mathrm{C}$ hyperfine interaction in $\mathrm{CO}_{2}^{--}$can be ignored at natural isotopic abundance $(1.1 \%)$. The initial state of the radical pair was taken to be a 1:3 mixture of singlet and triplet. The calculations involving isotopologues were identical except for the electron-nuclear interactions. The hyperfine coupling in the deuterium atom is smaller than $a_{\mathrm{H}}$ by the ratio of the magnetogyric ratios of the 
two nuclei: $a_{\mathrm{D}}=\left(\gamma_{\mathrm{D}} / \gamma_{\mathrm{H}}\right) a_{\mathrm{H}}=7.79 \mathrm{mT}$ and the isotropic ${ }^{13} \mathrm{C}$ hyperfine coupling in ${ }^{13} \mathrm{CO}_{2}^{\bullet-}$ is $a_{\mathrm{C}}$ $=19.93 \mathrm{mT}^{27}$

The reaction steps shown in Fig. 1(a) were modelled using a Haberkorn superoperator, $\mathbf{K},{ }^{28}$ for the spin-selective formation of formic acid from the singlet radical pair (with a first-order rate constant, $k_{\mathrm{S}}$ ) and by allowing the radicals in both singlet and triplet states to diffuse apart (with the same first-order rate constant, $k_{\mathrm{D}}$ ):

$$
\mathbf{K}=\frac{1}{2} k_{\mathrm{S}}\left(P^{\mathrm{S}} \otimes E+E \otimes P^{\mathrm{S}}\right)+k_{\mathrm{D}} E \otimes E
$$

where $P^{\mathrm{S}}$ is the singlet projection operator and $E$ is the identity operator. $k_{\mathrm{S}}$ and $k_{\mathrm{D}}$ were treated as variable parameters.

It has long been established that electron spin relaxation can have profound effects on the sensitivity of radical pair reactions to applied magnetic fields. ${ }^{29-32}$ Spin relaxation in $\mathrm{CO}_{2}^{--}$was modelled by one of three mechanisms: (1) generic "random fields" relaxation of the three Cartesian components of the electron spin; ${ }^{33}(2)$ singlet-triplet dephasing arising from diffusional modulation of the electron exchange interaction; ${ }^{23}$ (3) $g$-tensor anisotropy, i.e. rotational modulation of the anisotropic electron Zeeman interaction. ${ }^{20}$ For (1) and (2), a phenomenological relaxation rate constant, $w$, was varied, along with $k_{\mathrm{S}}$ and $k_{\mathrm{D}}$, in an attempt to match the calculated yield of formate to the experimental data. For (3), the variable parameter was the rotational correlation time, $\tau_{\mathrm{c}}$, of $\mathrm{CO}_{2}^{--}$. The principal components of the $g$-tensor of $\mathrm{CO}_{2}^{--}$, required for mechanism (3), were: $g_{x x}=2.0032 ; g_{y y}=1.9975 ; g_{z z}=2.0014 .{ }^{26}$ The three relaxation superoperators were:

$$
\begin{gathered}
\mathbf{W}^{(1)}=w\left[\frac{3}{4} E \otimes E-S_{\mathrm{A} x} \otimes \tilde{S}_{\mathrm{A} x}-S_{\mathrm{A} y} \otimes \tilde{S}_{\mathrm{A} y}-S_{\mathrm{A} z} \otimes \tilde{S}_{\mathrm{A} z}\right], \\
\mathbf{W}^{(2)}=w\left[P^{\mathrm{S}} \otimes P^{\mathrm{T}}+P^{\mathrm{T}} \otimes P^{\mathrm{S}}\right],
\end{gathered}
$$




$$
\begin{gathered}
\mathbf{W}^{(3)}=\frac{1}{15} \sum_{j=x, y, z}\left(\frac{g_{j j}-g_{\text {iso }}}{g_{\text {iso }}}\right)^{2} \omega_{0}^{2} \\
\times\left[3 J\left(\omega_{0}\right)\left(\frac{1}{2} E \otimes E-S_{\mathrm{A} x} \otimes \tilde{S}_{\mathrm{A} x}-S_{\mathrm{A} y} \otimes \tilde{S}_{\mathrm{A} y}\right)+2 J(0)\left(\frac{1}{2} E \otimes E-2 S_{\mathrm{A} z} \otimes \tilde{S}_{\mathrm{A} z}\right)\right] .
\end{gathered}
$$

$S_{\mathrm{Aj}}(j=x, y, z)$ are the components of the electron spin operator of $\mathrm{CO}_{2}^{\bullet-}$, the tilde $(\sim)$ indicates the operator transpose, $P^{\mathrm{S}}$ and $P^{\mathrm{T}}$ are the singlet and triplet projection operators, $g_{\text {iso }}=\left(g_{x x}+g_{y y}+g_{z z}\right) / 3$ is the isotropic $g$-value of $\mathrm{CO}_{2}^{\bullet-}, \omega_{0}=g_{\text {iso }} \mu_{\mathrm{B}} B / \hbar$ is the electron Larmor frequency, and the spectral density function is taken to be

$$
J(\omega)=\frac{\tau_{\mathrm{c}}}{1+\omega^{2} \tau_{\mathrm{c}}^{2}}
$$

Only the $g$-anisotropy mechanism, Eq. (4), depends explicitly on the applied magnetic field, with relaxation expected to be faster at higher fields. Electron spin relaxation in $\mathrm{H}^{\bullet}$ was assumed to be negligible.

The superoperators for the coherent spin dynamics (spin Hamiltonian $H$ ), the reactivity of the radicals and their spin relaxation were combined into a Liouvillian, $\mathbf{L}$,

$$
\mathbf{L}=i(H \otimes E-E \otimes \tilde{H})+\mathbf{K}+\mathbf{W}^{(k)}
$$

from which the fractional yield of formate, $\Phi_{\mathrm{S}}$ was calculated (see Supplementary Material) as

$$
\Phi_{\mathrm{S}}=\left(\frac{k_{\mathrm{S}}}{4 Z}\right) P^{\mathrm{S}} \cdot \mathbf{L}^{-1} \cdot E
$$

where $Z$ is the total number of nuclear spin states.

\section{RESULTS}

\section{Hyperfine and $\Delta g$ mechanisms}

We start by exploring whether it is possible to account qualitatively for the increased yield of formate in the presence of an applied magnetic field of up to $900 \mathrm{mT}$. Many of the simulations 
performed in this study predict a pronounced "low field effect" $34-36$ visible as a sharp rise in $\Phi_{\mathrm{S}}$ for weak magnetic fields, on the order of a few millitesla. Pan et al. did not report such a feature and might have overlooked it had it been present. ${ }^{8}$ We therefore excluded fields weaker than 30 $\mathrm{mT}$ from our attempts to match the simulations to the experimental data. We define magnetic field effects (MFEs) as positive or negative, according to whether the product yield, $\Phi_{\mathrm{S}}$, at $900 \mathrm{mT}$ is larger or smaller than that at $30 \mathrm{mT}$. As mentioned above, Pan et al. observed a positive MFE and attributed it to a dominant $\Delta g$ mechanism. ${ }^{8}$

Initial simulations were performed without spin relaxation, varying the rate constants $k_{\mathrm{s}}$ and $k_{\mathrm{D}}$ over a wide range from $10^{3}$ to $10^{11} \mathrm{~s}^{-1}$. The upper limit was chosen as follows. For there to be a significant magnetic field effect on the $\mathrm{H}^{\bullet}+\mathrm{CO}_{2}^{\bullet-}$ reaction, the exchange interaction of the two radicals must not greatly exceed the $\mathrm{H}^{\bullet}$ hyperfine coupling, meaning that the diffusing radicals must, on average, be more than 1.0 to $1.5 \mathrm{~nm}$ apart. An estimate of the upper limit on $k \mathrm{~s}$ is therefore the reciprocal of the time required for one or both of the radicals to diffuse $\Delta r \approx 1 \mathrm{~nm}$ from this average separation to the contact distance at which they are able to combine to produce $\mathrm{HCO}_{2}^{-}$. The translational diffusion coefficients of small molecules in water at room temperature are $<\sim 10^{-9} \mathrm{~m}^{2} \mathrm{~s}^{-1}$, so that $k_{\mathrm{S}} \approx D / \Delta r^{2}<10^{9} \mathrm{~s}^{-1}$. The magnetic field effect will also be very small if $k_{\mathrm{D}}$ greatly exceeds the frequency of singlet-triplet interconversion, $\gamma_{\mathrm{e}} a_{\mathrm{H}} \approx 9 \times 10^{9} \mathrm{~s}^{-1}$. We therefore set the upper limit for both rate constants at $10^{11} \mathrm{~s}^{-1}$.

When the hyperfine interaction in the hydrogen atom was omitted (i.e. $a_{\mathrm{H}}=0$ ), so that the $\Delta g$ mechanism was the only source of singlet-triplet interconversion, the MFE was found to be positive for all combinations of rate constants within the above range. However, when the hyperfine interaction was reinstated and the two $g$-values replaced by their mean (i.e. $\Delta g=0$ ), so that the field-dependence came solely from the hyperfine mechanism, the MFE was always negative. Illustrative simulations can be found in the Supplementary Material. When both mechanisms operated simultaneously (i.e. $a_{\mathrm{H}}=50.74 \mathrm{mT}$ and $\Delta g=0.0107$ ), the MFE remained negative over the whole range of $k_{\mathrm{S}}$ and $k_{\mathrm{D}}$ values. Contrary to the suggestion of Pan et al., ${ }^{8}$ the $\Delta g$ mechanism is outweighed by the hyperfine mechanism and the two, acting together, cannot 
account for the observed change in the magneto-current. This can be understood in terms of the faster singlet-triplet mixing coming from the hyperfine mechanism, $\gamma_{\mathrm{e}} a_{\mathrm{H}} / 2 \pi \approx 1420 \mathrm{MHz}$, compared to the $\Delta g$ mechanism, $\Delta g \mu_{\mathrm{B}} B / h \approx 135 \mathrm{MHz}$ (when $B=900 \mathrm{mT}$ ). As there are no other time-independent magnetic interactions in either $\mathrm{H}^{\bullet}$ or $\mathrm{CO}_{2}^{--}$that could reverse the dominance of the hyperfine mechanism, it seems likely that electron spin-relaxation is important. This conclusion is supported by the fact that the magneto-current is still increasing at $900 \mathrm{mT}$ (Fig. 1(b)). ${ }^{8}$ Without significant spin relaxation, the magnetic field effect should start to saturate as soon as the field exceeds the $\sim 50 \mathrm{mT}$ hyperfine interaction. ${ }^{15,37}$

\section{Phenomenological spin relaxation}

To assess whether random time-dependent local fields (Eq. (2)) or singlet-triplet dephasing (Eq. (3)) could give rise to positive MFEs, we simulated $\Phi_{\mathrm{S}}$ for relaxation rate constants, $w$, between $10^{5}$ and $10^{9} \mathrm{~s}^{-1}$, and $k \mathrm{~s}$ and $k_{\mathrm{D}}$ in the range $10^{3}$ to $10^{11} \mathrm{~s}^{-1}$, with $a_{\mathrm{H}}=50.74 \mathrm{mT}$ and $\Delta g=0.0107$, as above. Although inclusion of each of these relaxation processes changed the shape of the fielddependence, the MFEs remained strictly negative. Illustrative simulations can be found in the Supplementary Material. This result is not surprising. In magnetic fields comparable to the hyperfine interaction, singlet-triplet interconversion is more efficient than at higher fields where the $T_{+1}$ and $T_{-1}$ triplet states are energetically isolated from the singlet state and therefore much slower to convert to singlet and subsequently react to give the product. Evidently, the two relaxation processes considered above, with their field-independent rate constants, do not qualitatively change that situation. We therefore speculate that a positive MFE on the $\mathrm{H}^{\bullet}+\mathrm{CO}_{2}^{\bullet-}$ reaction might arise from a relaxation process that accelerates as the strength of the magnetic field, $B$, is increased. More efficient spin-lattice relaxation of the initial population of $\mathrm{T}_{+1}$ and $\mathrm{T}_{-1}$ triplets to the $\mathrm{T}_{0}$ state at high field should boost formation of the singlet radical pair state and hence the yield of $\mathrm{HCO}_{2}^{-}$.

To explore this possibility, we allowed the rate constant in the random fields relaxation superoperator, Eq. (2), to increase quadratically with the magnetic field: $w=q B^{2}$. By varying the proportionality constant $q$ between $10^{5}$ and $10^{10} \mathrm{~T}^{-2} \mathrm{~s}^{-1}$, and $k_{\mathrm{S}}$ and $k_{\mathrm{D}}$ between $10^{3}$ and $10^{11} \mathrm{~s}^{-1}$, we did indeed find combinations of the three parameters that gave positive MFEs. 


\section{g-tensor anisotropy relaxation}

Encouraged by the last result, we turned to the $g$-tensor anisotropy mechanism, Eq. (4), in which the field-dependence of the relaxation superoperator can be derived from the properties of the $\mathrm{CO}_{2}^{\circ-}$ radical. Once again, $\Phi_{\mathrm{S}}$ was calculated using $a_{\mathrm{H}}=50.74 \mathrm{mT}$ and $\Delta g=0.0107$. Agreement between the predictions of the model and the experimental data were sought by optimising the value of $\tau_{\mathrm{c}}$, for integer values of $\log _{10}\left(k_{\mathrm{S}}\right)$ and $\log _{10}\left(k_{\mathrm{D}}\right)$ between 3 and 11 , using a least-squares fitting procedure. Simulations of

$$
\frac{\Phi_{\mathrm{S}}(B)-\Phi_{\mathrm{S}}(30 \mathrm{mT})}{\left|\Phi_{\mathrm{S}}(900 \mathrm{mT})-\Phi_{\mathrm{S}}(30 \mathrm{mT})\right|}
$$

were compared with the experimental data (Fig. 1(b)). Satisfactory agreement was found for 17 sets of the three parameters, summarised in Table 1. Four of them correspond to short-lived radical pairs with long rotational correlation times: $k_{\mathrm{S}}=10^{10}$ or $10^{11} \mathrm{~s}^{-1}, k_{\mathrm{D}}=10^{9}, 10^{10}$ or $10^{11} \mathrm{~s}^{-1}$, and $\tau_{\mathrm{c}} \geq 1 \mu \mathrm{s}$. The remainder have $10^{6}<k_{\mathrm{S}}<10^{11} \mathrm{~s}^{-1}, 10^{3}<k_{\mathrm{D}}<10^{5} \mathrm{~s}^{-1}$, and $\tau_{\mathrm{c}}$ between $\sim 1 \mathrm{ps}$ and 15 ps. We discuss the interpretation of these results in the following section.

\begin{tabular}{cc|cccccc} 
& & \multicolumn{7}{c}{$\log _{10}(k \mathrm{~s})$} \\
& & 6 & 7 & 8 & 9 & 10 & 11 \\
\cline { 2 - 8 } & 3 & 1.25 & 0.61 & 0.61 & 0.64 & 0.65 & 0.65 \\
4 & - & 8.9 & 4.6 & 5.4 & 5.7 & 5.7 \\
$\log _{10}\left(k_{\mathrm{D}}\right)$ & 5 & - & - & - & - & 12.4 & 14.2 \\
& $6-8$ & - & - & - & - & - & - \\
& 9 & - & - & - & - & - & $1.0^{*}$ \\
10 & - & - & - & - & $10.3^{*}$ & $9.1^{*}$ \\
& 11 & - & - & - & - & - & $65^{*}$
\end{tabular}

Table 1. Optimised values of the rotational correlation time, $\tau_{\mathrm{c}}$, for combinations of rate constants $k \mathrm{~s}$ and $k_{\mathrm{D}}\left(\right.$ in $^{-1}$ ) that predict magnetic field effects in agreement with the experimental data of Pan et al. ${ }^{8}$ The units of $\tau_{\mathrm{c}}$ are picoseconds apart from the asterisked entries which are in microseconds. Spin relaxation was modelled by means of the $g$-tensor anisotropy mechanism. Missing entries in 
the table indicate combinations of $k_{\mathrm{S}}$ and $k_{\mathrm{D}}$ for which satisfactory fits to the data could not be obtained. The shaded area highlights the most credible values of the three parameters (see below).

Figure 2 shows the magnetic field effect on $\left[{ }^{1} \mathrm{H}^{\bullet}{ }^{12} \mathrm{CO}_{2}^{\bullet-}\right]$ (and its isotopologues, discussed below) calculated for two of the sets of parameter values in Table 1. Like the other entries in Table 1, both show satisfactory agreement with the (scaled) magneto-current data.

\section{Spin-rotation relaxation}

A second relaxation mechanism that depends on the electronic $g$-tensor arises from the interaction of the electron spin with the rotational angular momentum of the radical. The relaxation superoperator for this mechanism has the same form as the random fields superoperator, Eq. (2), with the rate constant, $w$, given by:

$$
w=\frac{1}{9 \tau_{\mathrm{c}}} \sum_{j=x, y, z}\left(g_{j j}-g_{\mathrm{e}}\right)^{2}
$$

where $g_{\mathrm{e}} \approx 2.0023$ is the free-electron $g$-value. ${ }^{21,22}$ With this mechanism in place of $g$-tensor anisotropy, the simulated MFEs were uniformly negative for $10^{3}<k_{\mathrm{s}}, k_{\mathrm{D}}<10^{11} \mathrm{~s}^{-1}$ and $1 \mathrm{ps}<\tau_{\mathrm{c}}$ $<1 \mu$ s. Apart from a few cases in which the MFEs were very small $(<1 \%)$, the same was true when the two relaxation mechanisms were allowed to operate simultaneously.

To summarise, we were able to satisfactorily model the magneto-current using $g$-tensor anisotropy as the spin relaxation mechanism but not with spin-rotation or with $g$-tensor anisotropy and spinrotation acting together.

\section{Isotopologous reactions}

Isotopic substitution affects radical pair reactions principally via the changes in hyperfine interactions associated with the different magnetic moments of the isotopes. ${ }^{38}$ In the case of $\mathrm{H}^{\bullet}+$ $\mathrm{CO}_{2}^{--} \rightarrow \mathrm{HCO}_{2}^{-}$, in which a covalent bond is formed in a reaction with little or no activation energy, mass isotope effects should be very small and magnetic isotope effects should dominate. 
Figures 2(a) and (b) show MFEs calculated for $\left[\mathrm{H}^{\bullet} \mathrm{CO}_{2}^{\bullet-}\right]$ radical pairs in which hydrogen has been replaced by deuterium, or ${ }^{12} \mathrm{C}$ by ${ }^{13} \mathrm{C}$, or both. Deuterium has a hyperfine coupling $\sim 6.5$ times smaller than hydrogen, and ${ }^{13} \mathrm{C}$ substitution in $\mathrm{CO}_{2}^{\circ-}$ introduces a hyperfine coupling of 19.93 mT. ${ }^{27}$ In Fig. 2(a), the MFEs of all three isotopologues of $\left[{ }^{1} \mathrm{H}^{\bullet}{ }^{12} \mathrm{CO}_{2}^{\bullet-}\right]$ are biphasic, initially decreasing and then increasing when $B>200-300 \mathrm{mT}$. For the set of parameters chosen for Fig. 2(b), the MFE remains monophasic and is significantly larger for ${ }^{2} \mathrm{H}^{\bullet}$ than for ${ }^{1} \mathrm{H}^{\bullet}$.

\section{DISCUSSION}

Table 1 summarises the results of our attempts to account for the magneto-current reported by Pan et al. ${ }^{8}$ including hyperfine and $\Delta g$ mechanisms of singlet-triplet interconversion in the radical pair, $\left[\mathrm{H}^{\bullet} \mathrm{CO}_{2}^{\bullet-}\right]$. Good agreement with the data was achieved for certain values of the three parameters, $k_{\mathrm{S}}, k_{\mathrm{D}}$ and $\tau_{\mathrm{c}}$, provided the dominant electron spin relaxation pathway in $\mathrm{CO}_{2}^{--}$is rotational modulation of the anisotropic Zeeman ( $g$-tensor) interaction. But which, if any, of the 17 entries in Table 1 are credible? A lower bound on $\tau_{\mathrm{c}}$ for $\mathrm{CO}_{2}^{--}$can be obtained from the Stokes-Einstein equation for the rotational diffusion of a sphere of radius $a$ in a medium of viscosity $\eta$ at temperature $T$,

$$
\tau_{\mathrm{c}}=\frac{4 \pi \eta a^{3}}{3 k_{\mathrm{B}} T} .
$$

Taking $a \approx 150 \mathrm{pm}$ for $\mathrm{CO}_{2}, \eta \approx 10^{-3} \mathrm{~kg} \mathrm{~m}^{-1} \mathrm{~s}^{-1}$ for water at $T=298 \mathrm{~K}$, Eq. (10) gives $\tau_{\mathrm{c}} \approx 3.4$ ps. Therefore we can probably discount the top row of Table $1\left(k_{\mathrm{D}}=10^{3} \mathrm{~s}^{-1}\right)$ in which $\tau_{\mathrm{c}} \approx 1 \mathrm{ps}$. The four entries in the bottom right hand corner of Table 1 are also improbable, for two reasons. First, the values of $k_{\mathrm{S}}\left(10^{10}\right.$ and $\left.10^{11} \mathrm{~s}^{-1}\right)$ exceed by some margin the above-estimated upper limit on the rate constant for production of formate, $D / \Delta r^{2} \approx 10^{9} \mathrm{~s}^{-1}$. Second, the expression used for the relaxation superoperator, Eq. (4), is unlikely to be valid for rotational correlation times as long as $1 \mu \mathrm{s}$ which do not satisfy the conditions for motional narrowing. It therefore seems reasonable to exclude the final two columns of Table 1. 
We are therefore left with the shaded area in Table 1, i.e. $\tau_{\mathrm{c}} \approx 5-10 \mathrm{ps}, k \mathrm{D} \approx 10^{4} \mathrm{~s}^{-1}, k \mathrm{~s} \approx 10^{7}$ $10^{9} \mathrm{~s}^{-1}$ as the conditions most likely to be realistic. The small value of $k_{\mathrm{D}}$ and the much larger value of $k$ s may be understood by reference to Fig. 3 which shows the field-dependence of the spinlattice relaxation rate of $\mathrm{CO}_{2}^{\circ-}$ predicted by the $g$-tensor anisotropy mechanism, ${ }^{19}$

$$
R=\frac{1}{5} \sum_{j=x, y, z}\left(\frac{g_{j j}-g_{\text {iso }}}{g_{\text {iso }}}\right)^{2}\left[\frac{\omega_{0}^{2} \tau_{\mathrm{c}}}{1+\omega_{0}^{2} \tau_{\mathrm{c}}^{2}}\right],
$$

with $\tau_{\mathrm{c}}=1,5$, and $25 \mathrm{ps}$. For all three correlation times relaxation is faster at higher field, as required for a positive MFE. Without efficient spin-lattice relaxation at high field, radical pairs initially in the $\mathrm{T}_{+1}$ and $\mathrm{T}_{-1}$ states (50\% of the total) can never produce $\mathrm{HCO}_{2}^{-}$. Strongly fielddependent spin-lattice relaxation of the kind shown in Fig. 3 transfers population from the unreactive $T_{+1}$ and $T_{-1}$ states to $T_{0}$ which mixes coherently with the singlet state which then gives $\mathrm{HCO}_{2}^{-}$. This process is most efficient when $\omega_{0} \tau_{\mathrm{c}} \approx 1$, i.e. $\tau_{\mathrm{c}} \approx 6.3$ ps and $R_{\max } \approx 6.7 \times 10^{4} \mathrm{~s}^{-1}$ when $B=900 \mathrm{mT}$. It is no coincidence, therefore, that this estimate of $\tau_{\mathrm{c}}$ falls within the range of $\tau_{\mathrm{c}}$ values in the shaded region of Table 1 . Nor is it surprising that $k_{\mathrm{D}}$ must be as slow as $\sim 10^{4} \mathrm{~s}^{-1}$ (Table 1): if $k_{\mathrm{D}}$ is not smaller than $R_{\max }$, the $\mathrm{T}_{+1}$ and $\mathrm{T}_{-1}$ pairs will separate before they can relax to $\mathrm{T}_{0}$.

The small value of $k_{\mathrm{D}}\left(\sim 10^{4} \mathrm{~s}^{-1}\right)$ suggests that both radicals may be loosely adsorbed on the surface of the catalyst: freely diffusing radicals would separate much faster than $10^{4} \mathrm{~s}^{-1}$. That $\tau_{\mathrm{c}}$ (Table 1) is only a little larger than the Stokes-Einstein estimate (3.4 ps) implies that the rotational motion of $\mathrm{CO}_{2}^{\bullet-}$ is not strongly constrained by adsorption.

Finally, we turn to the magnetic isotope effects in Fig. 2(a). The pronounced qualitative changes in the predicted magnetic field effects could form the basis of an experimental test of the reaction mechanism proposed by Pan et al. ${ }^{8}$ and of the model used here to interpret their data. 


\section{CONCLUSIONS}

We have used radical pair spin dynamics simulations to interpret the effects of applied magnetic fields on the electrocatalytic reduction of $\mathrm{CO}_{2}$ to $\mathrm{HCO}_{2}^{-}$reported by Pan et al. ${ }^{8}$ Our main conclusions are: (1) The $\Delta g$ mechanism favoured by Pan et al. ${ }^{8}$ cannot, on its own, account for the observed magnetic field effects. (2) The observed magneto-current is consistent with fielddependent spin relaxation in $\mathrm{CO}_{2}^{--}$combined with coherent singlet-triplet mixing driven by the isotropic hyperfine and Zeeman interactions in $\mathrm{H}^{\bullet}$ and $\mathrm{CO}_{2}^{--}$. Our analysis puts strong constraints on the parameters of the model. (3) Isotopic substitution could be used to test both the proposed reaction mechanism, ${ }^{8}$ Fig. 1(a), and the model presented here.

\section{SUPPLEMENTARY MATERIAL}

See Supplementary Material for details of the spin dynamics calculations and additional simulations of magnetic field effects.

\section{DATA AVAILABILITY}

Data sharing is not applicable to this article as no new data were created or analysed in this study.

\section{ACKNOWLEDGEMENTS}

This work was supported in part by the Air Force Office of Scientific Research (Air Force Materiel Command, USAF award no. FA9550-14-1-0095). 


\section{FIGURES}

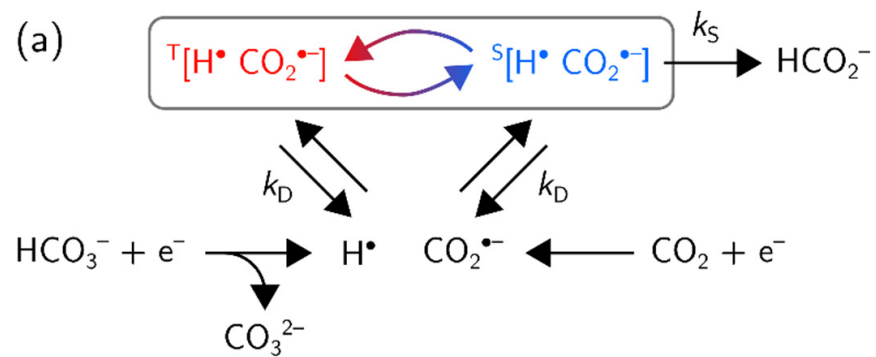

(b)

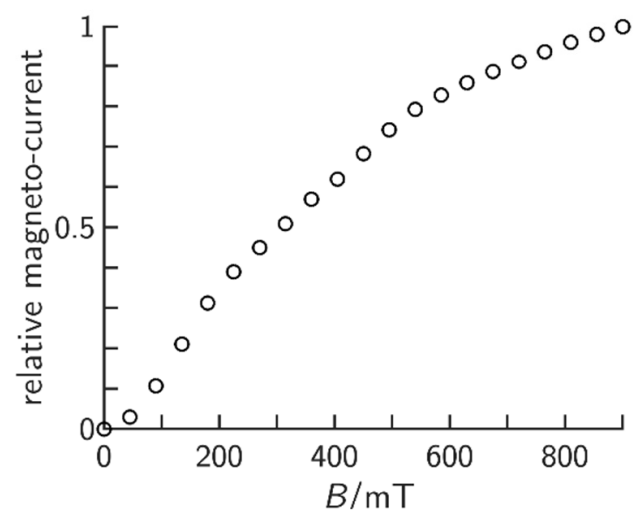

FIG. 1. (a) Proposed reaction scheme for the electrocatalytic reduction of carbon dioxide to formic acid. $^{8}$ (b) Dependence of the magneto-current on the applied magnetic field constructed from the time-dependence of these two quantities depicted in Fig. 2(b) of Pan et al. ${ }^{8}$ The field-dependence shown here is that reported for a $0.3 \mathrm{M}$ bicarbonate solution, rescaled to 1.0 at $0.9 \mathrm{~T}$. The data recorded from $0.1 \mathrm{M}$ and $0.2 \mathrm{M}$ solutions have essentially identical shapes. Pan et al. have reported similar magnetic field effects for other chemical systems. ${ }^{39,40}$ 

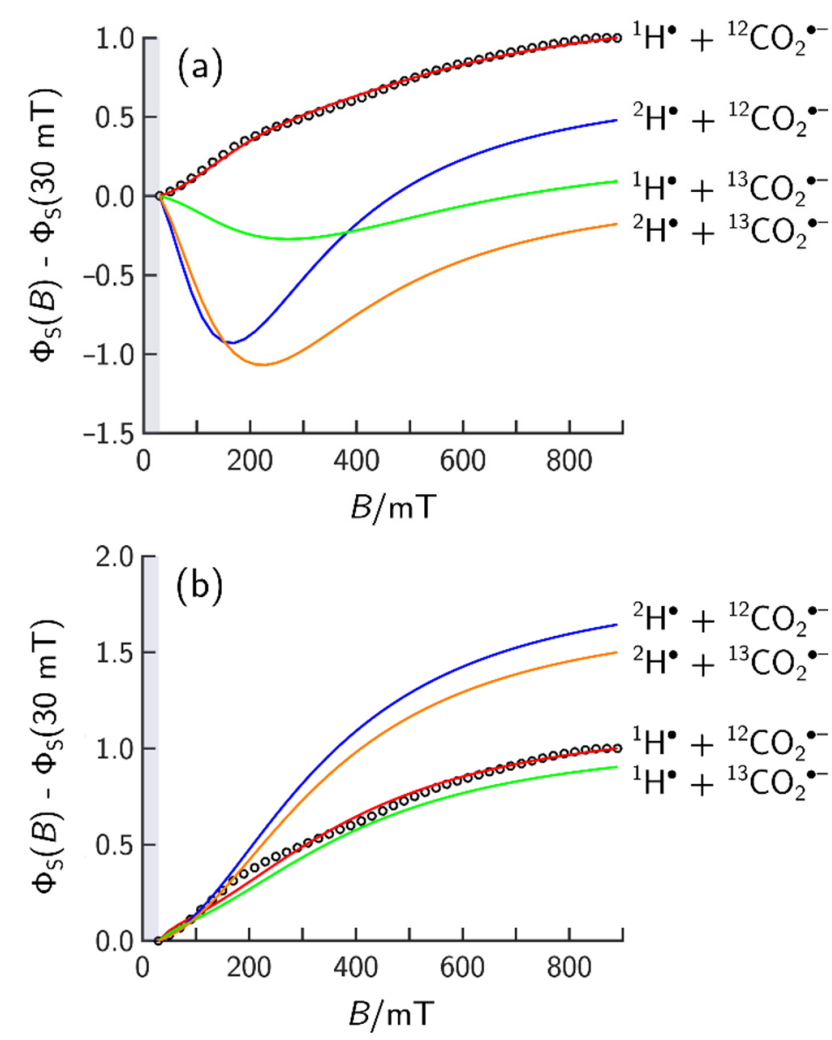

FIG. 2. Calculated magnetic field effects on the $\mathrm{H}^{\bullet}+\mathrm{CO}_{2}^{\bullet-}$ reaction with spin-relaxation arising from the $g$-tensor anisotropy mechanism, Eq. (4). (a) $k_{\mathrm{s}}=10^{7} \mathrm{~s}^{-1}, k_{\mathrm{D}}=10^{4} \mathrm{~s}^{-1}, \tau_{\mathrm{c}}=8.9 \mathrm{ps}$. (b) $k \mathrm{~s}$ $=10^{11} \mathrm{~s}^{-1}, k_{\mathrm{D}}=10^{9} \mathrm{~s}^{-1}, \tau_{\mathrm{c}}=1.0 \mu \mathrm{s}$. The graphs show the difference between the reaction yield at field $B$ and that at $30 \mathrm{mT}$, scaled to 1.0 at $900 \mathrm{mT}$ for ${ }^{1} \mathrm{H}^{\bullet}+{ }^{12} \mathrm{CO}_{2}^{\bullet-}$. In each panel, the same scaling factor was used for the four isotopologues. The open circles show the measured magnetocurrent. ${ }^{8}$ The corresponding plots showing the low field effects $(B<30 \mathrm{mT})$ can be found in the Supplementary Material. Although giving a satisfactory fit to the experimental magneto-current, the parameters required for (b) are unlikely to be realistic (see Discussion section). 


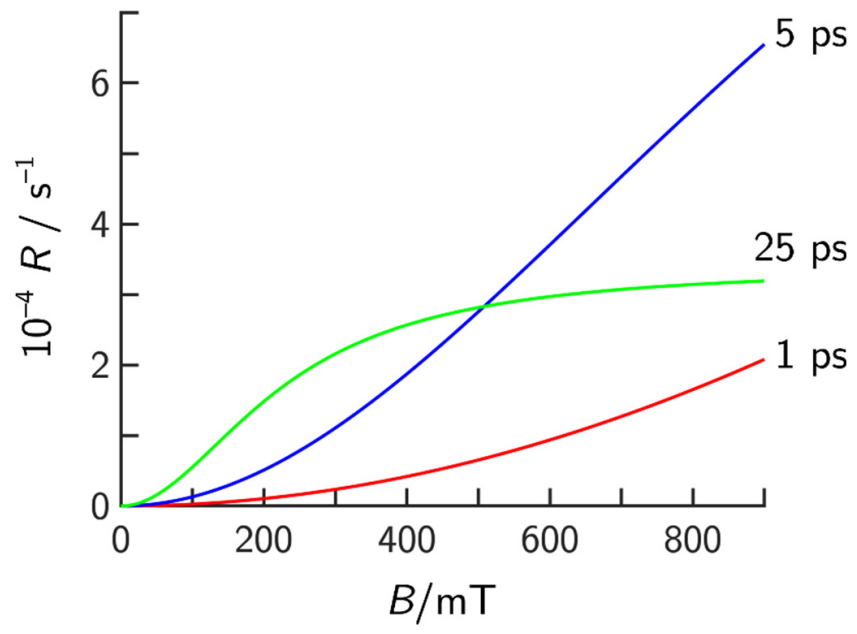

FIG. 3. Calculated spin-lattice relaxation rate, Eq. (11), of $\mathrm{CO}_{2}^{\bullet-}$ ( $g$-tensor anisotropy mechanism) plotted as a function of the magnetic field strength, for $\tau_{\mathrm{c}}=1,5$ and $25 \mathrm{ps}$. 


\section{REFERENCES}

${ }^{1}$ W. Wang, S. P. Wang, X. B. Ma and J. L. Gong, Chem. Soc. Rev. 40, 3703 (2011).

${ }^{2}$ E. V. Kondratenko, G. Mul, J. Baltrusaitis, G. O. Larrazabal and J. Perez-Ramirez, Energy Environ. Sci. 6, 3112 (2013).

${ }^{3}$ A. M. Appel, J. E. Bercaw, A. B. Bocarsly, H. Dobbek, D. L. DuBois, M. Dupuis, J. G. Ferry, E. Fujita, R. Hille, P. J. A. Kenis, C. A. Kerfeld, R. H. Morris, C. H. F. Peden, A. R. Portis, S. W. Ragsdale, T. B. Rauchfuss, J. N. H. Reek, L. C. Seefeldt, R. K. Thauer and G. L. Waldrop, Chem. Rev. 113, 6621 (2013).

${ }^{4}$ S. N. Habisreutinger, L. Schmidt-Mende and J. K. Stolarczyk, Angew. Chem. 52, 7372 (2013). ${ }^{5}$ M. Aresta, A. Dibenedetto and A. Angelini, Chem. Rev. 114, 1709 (2014).

${ }^{6}$ G. A. Ozin, Adv. Mater. 27, 1957 (2015).

${ }^{7}$ D. D. Zhu, J. L. Liu and S. Z. Qiao, Adv. Mater. 28, 3423 (2016).

${ }^{8}$ H. P. Pan, X. X. Jiang, X. K. Wang, Q. L. Wang, M. K. Wang and Y. Shen, J. Phys. Chem. Lett. 11, 48 (2020).

${ }^{9}$ U. E. Steiner and T. Ulrich, Chem. Rev. 89, 51 (1989).

${ }^{10}$ D. E. Manolopoulos and P. J. Hore, J. Chem. Phys. 139, 124106 (2013).

${ }^{11}$ B. Brocklehurst, Chem. Soc. Rev. 31, 301 (2002).

${ }^{12}$ P. J. Hore and H. Mouritsen, Annu. Rev. Biophys. 45, 299 (2016).

${ }^{13}$ C. T. Rodgers, Pure Appl. Chem. 81, 19 (2009).

${ }^{14}$ A. Lewis, Spin Dynamics in Radical Pairs (Springer International Publishing, 2018).

${ }^{15}$ K. Maeda, A. J. Robinson, K. B. Henbest, H. J. Hogben, T. Biskup, M. Ahmad, E. Schleicher, S. Weber, C. R. Timmel and P. J. Hore, Proc. Natl. Acad. Sci. USA 109, 4774 (2012).

${ }^{16}$ K. M. Salikhov, Y. N. Molin, R. Z. Sagdeev and A. L. Buchachenko, Spin Polarization and Magnetic Field Effects in Radical Reactions (Elsevier, New York, 1984).

${ }^{17}$ K. A. McLauchlan and U. E. Steiner, Mol. Phys. 73, 241 (1991).

${ }^{18}$ T. Y. Karogodina, I. G. Dranov, S. V. Sergeeva, D. V. Stass and U. E. Steiner, Chem. Phys. Chem. 12, 1714 (2011).

${ }^{19}$ N. M. Atherton, Principles of Electron Spin Resonance (Ellis Horwood, Chichester, 1993).

${ }^{20}$ D. Kivelson, J. Chem. Phys. 33, 1094 (1960).

${ }^{21}$ G. Nyberg, Molec. Phys. 12, 69 (1967).

${ }^{22}$ P. W. Atkins and D. Kivelson, J. Chem. Phys. 44, 169 (1966). 
${ }^{23}$ A. I. Shushin, Chem. Phys. Lett. 181, 274 (1991).

${ }^{24}$ A. Carrington and A. D. McLachlan, Introduction to Magnetic Resonance (Harper \& Row, New York, 1967).

${ }^{25}$ K. Ishikawa, N. Sumi, A. Kono, H. Horibe, K. Takeda, H. Kondo, M. Sekine and M. Hori, J. Phys. Chem. Lett. 2, 1278 (2011).

${ }^{26}$ D. W. Ovenall and D. H. Whiffen, Molec. Phys. 4, 135 (1961).

${ }^{27}$ J. H. Lunsford and J. P. Jayne, J. Phys. Chem. 69, 2182 (1965).

${ }^{28}$ R. Haberkorn, Mol. Phys. 32, 1491 (1976).

${ }^{29}$ H. Hayashi and S. Nagakura, Bull. Chem. Soc. Jpn. 57, 322 (1984).

${ }^{30}$ R. Nakagaki, M. Yamaoka, O. Takahira, K. Hiruta, Y. Fujiwara and Y. Tanimoto, J. Phys. Chem. A 101, 556 (1997).

${ }^{31}$ T. Klumpp, M. Linsenmann, S. L. Larson, B. R. Limoges, D. Burssner, E. B. Krissinel, C. M. Elliott and U. E. Steiner, J. Am. Chem. Soc. 121, 1076 (1999).

${ }^{32}$ H. Hayashi, Y. Sakaguchi and M. Wakasa, Bull. Chem. Soc. Jpn. 74, 773 (2001).

${ }^{33}$ D. R. Kattnig, J. K. Sowa, I. A. Solov'yov and P. J. Hore, New J. Phys. 18, 063007 (2016).

${ }^{34}$ B. Brocklehurst, J. Chem. Soc. Faraday Trans. II 72, 1869 (1976).

${ }^{35}$ U. Till, C. R. Timmel, B. Brocklehurst and P. J. Hore, Chem. Phys. Lett. 298, 7 (1998).

${ }^{36}$ C. R. Timmel, U. Till, B. Brocklehurst, K. A. McLauchlan and P. J. Hore, Molec. Phys. 95, 71 (1998).

${ }^{37}$ T. Miura, K. Maeda and T. Arai, J. Phys. Chem. A 110, 4151 (2006).

${ }^{38}$ K. M. Salikhov, Magnetic Isotope Effect in Radical Reactions (Springer-Verlag, Vienna, 1996).

${ }^{39}$ H. P. Pan, X. Xiao, B. Hu, Y. Shen and M. K. Wang, J. Phys. Chem. C 121, 28420 (2017).

${ }^{40}$ H. P. Pan, M. K. Wang, Y. Shen and B. Hu, J. Phys. Chem. C 122, 19880 (2018). 